Homology, Homotopy and Applications, vol.13(2), 2011, pp.37-42

\title{
A GENERALIZATION OF THE WANG SEQUENCE
}

\author{
HAIBAO DUAN
}

(communicated by Don Davis)

\begin{abstract}
The classical Wang sequence (for cohomology of fiber bundles over a sphere) is extended to a more generalized setting, given by gluing together two disc bundles over manifolds along their boundaries.
\end{abstract}

\section{The result}

Given an Euclidean vector bundle $\xi$ over a manifold $N$ let $D(\xi)$ and $S(\xi)=\partial D(\xi)$ be the unit disc bundle and the unit sphere bundle of $\xi$, respectively. That is,

$$
D(\xi)=\left\{v \in \xi \mid\|v\|^{2} \leqslant 1\right\}, S(\xi)=\left\{v \in \xi \mid\|v\|^{2}=1\right\} .
$$

A smooth manifold $M$ is said to have focal genus 2 if it admits a decomposition of the form

$$
M=D\left(\xi_{1}\right) \cup_{g} D\left(\xi_{2}\right),
$$

where $\xi_{t}$ is an oriented Euclidean vector bundle on a manifold $N_{t}, t=1,2$, and where $g: S\left(\xi_{2}\right) \rightarrow S\left(\xi_{1}\right)$ is a diffeomorphism. We identify each $N_{t}$ as a submanifold of $M$ via the obvious embedding $i_{t}: N_{t} \stackrel{\sigma_{t}}{\rightarrow} D\left(\xi_{t}\right) \subset M$, and call it a focal submanifold of $M$, where $\sigma_{t}$ is the zero section of the bundle $\xi_{t}$.

Let $p_{t}: S\left(\xi_{t}\right) \rightarrow N_{t}$ be the bundle projection, and put

$$
g_{t}= \begin{cases}g: S\left(\xi_{2}\right) \rightarrow S\left(\xi_{1}\right) & \text { for } t=1 ; \\ g^{-1}: S\left(\xi_{1}\right) \rightarrow S\left(\xi_{2}\right) & \text { for } t=2\end{cases}
$$

For each $t \in\{1,2\}$ let $\bar{t}$ be its complement in $\{1,2\}$.

In this paper all homologies and cohomologies are over integer coefficients. For a manifold $N$ write $1 \in H^{0}(N)$ for the multiplicative unit. Our main result is

Theorem 1.1. Let $M$ be a manifold having focal genus 2 and with focal submanifolds $N_{t}, t=1,2$. For each $t \in\{1,2\}$ there is an exact sequences

$$
\cdots \rightarrow H^{r-1}\left(N_{t}\right) \stackrel{\theta_{t}}{\rightarrow} H^{r-\operatorname{dim} \xi_{\bar{t}}}\left(N_{\bar{t}}\right) \stackrel{\alpha_{t}}{\rightarrow} H^{r}(M) \stackrel{i_{t}^{*}}{\rightarrow} H^{r}\left(N_{t}\right) \stackrel{\theta_{t}}{\rightarrow} \cdots,
$$

where

i) $\alpha_{t}\left(i_{\bar{t}}^{*}(x) \cup y\right)=x \cup \alpha_{t}(y)$ for $x \in H^{*}(M), y \in H^{*}\left(N_{\bar{t}}\right)$;

The author's research was supported by 973 Program 2011CB302400 and NSFC 11131008.

Received March 17, 2011, revised June 11, 2011; published on September 19, 2011.

2000 Mathematics Subject Classification: 55N10, 57N65.

Key words and phrases: Wang sequence, Gysin sequence, Thom isomorphism.

Article available at http://intlpress.com/HHA/v13/n2/a3 and doi:10.4310/HHA.2011.v13.n2.a3

Copyright (C) 2011, International Press. Permission to copy for private use granted. 
ii) $\theta_{t}=\beta_{\bar{t}} \circ g_{t}^{*} \circ p_{t}^{*}$ with $\beta_{t}: H^{*}\left(S\left(\xi_{t}\right)\right) \rightarrow H^{*}\left(N_{t}\right)$ the connecting homomorphism in the Gysin sequence of the fibration $p_{t}[\boldsymbol{8}, p .143]$.

Moreover, if both $N_{1}, N_{2}$ are oriented, and if $g$ is orientation reversing, then with respect to the induced orientation on $M$

iii) $\alpha_{t}(1)$ is the Poincare dual of the cycle class $i_{\bar{t} *}\left[N_{\bar{t}}\right] \in H_{*}(M)$.

The decomposition for $\theta_{t}$ in ii) is useful to derive its multiplicative properties. To explain this we examine the case where the oriented bundle $\xi_{2}$ has a decomposition $\xi_{2}=\eta \oplus \varepsilon$ with $\varepsilon$ the trivial $\mathbb{R}$-bundle on $N_{2}$. Let $s: N_{2} \rightarrow S\left(\xi_{2}\right)=S(\eta \oplus \varepsilon)$ be the section defined by $s(b)=(0,1)$, and write $i_{b}: p_{2}^{-1}(b) \rightarrow S\left(\xi_{2}\right)$ for the inclusion of the fibre sphere $p_{2}^{-1}(b), b \in N_{2}$. It is shown in [3] that there is a unique class $\alpha \in H^{\operatorname{dim} \xi_{2}-1}\left(S\left(\xi_{2}\right)\right)$ such that $s^{*}(\alpha)=0$ and $\left\langle i_{b}^{*}(\alpha),\left[p_{2}^{-1}(b)\right]\right\rangle=1, b \in N_{2}$. Moreover,

Lemma 1.2. ([3, Lemma 4]). As an $H^{*}\left(N_{2}\right)$-module, $H^{*}\left(S\left(\xi_{2}\right)\right)$ has the basis $\{1, \alpha\}$ subject to the single relation

$$
\alpha^{2}+e(\eta) \alpha=0
$$

where $e(\eta) \in H^{*}\left(N_{2}\right)$ is the Euler class of the oriented bundle $\eta$.

With respect to the $H^{*}\left(N_{2}\right)$-module structure on $H^{*}\left(S\left(\xi_{2}\right)\right)$ the composed ring map

$$
H^{*}\left(N_{1}\right) \stackrel{p^{*}}{\rightarrow} H^{*}\left(S\left(\xi_{1}\right)\right) \stackrel{g_{\rightarrow}^{*}}{\rightarrow} H^{*}\left(S\left(\xi_{2}\right)\right)=H^{*}\left(N_{2}\right)\{1, \alpha\}
$$

gives rise to two additive maps $\varphi, \psi: H^{*}\left(N_{1}\right) \rightarrow H^{*}\left(N_{2}\right)$ characterized by

$$
g_{1}^{*} \circ p_{1}^{*}(x)=\varphi(x)+\psi(x) \alpha, x \in H^{*}\left(N_{1}\right) .
$$

Corollary 1.3. Let $M=D\left(\xi_{1}\right) \cup_{g} D\left(\xi_{2}\right)$ be a manifold having focal genus 2 and with $\xi_{2}=\eta \oplus \varepsilon$. Then

$$
\theta_{1}=\psi: H^{*}\left(N_{1}\right) \rightarrow H^{*}\left(N_{2}\right)
$$

In particular

$$
\theta_{1}(x y)=\varphi(x) \theta_{1}(y)+(-1)^{(k-1)|y|} \theta_{1}(x) \varphi(y)+(-1)^{(k-1)|y|-k^{2}} \theta_{1}(x) \theta_{1}(y) e(\eta) .
$$

Remark 1.4. The author is grateful to Fuquan Fang and Zizhou Tang for informing him that a manifold $M=D\left(\xi_{1}\right) \cup_{g} D\left(\xi_{2}\right)$ with focal genus 2 coincides with the notion of the double mapping cylinder of the pairs of maps $p_{1}: S\left(\xi_{1}\right) \rightarrow N_{1}, p_{2} g_{2}: S\left(\xi_{1}\right) \rightarrow$ $N_{2}$ due to Grove and Halperin [4]. Letting $F$ be a path component of the homotopy fiber of the inclusion $S\left(\xi_{1}\right) \subset M$, then a classification on $F$ up to fundamental group, integral cohomology and rational homotopy type has been obtained by Grove and Halperin in [4, Theorem D].

The name "double mapping cylinder" could indicate the much more general construction of the adjoint space $M_{f} \cup M_{g}$, where $M_{f}$ and $M_{g}$ are the mapping cylinders of two maps $f: X \rightarrow Y$ and $g: X \rightarrow Z$ between topological spaces, and where the union is over the common subspace $X \times 1 \subset M_{f}, M_{g}$. With the term "focal genus 2 " we hope to emphasize the geometric decomposition (1) on $M$ given by two disc bundles over the focal submanifolds $N_{t}, t=1,2$. 


\section{Examples and applications}

Manifolds having focal genus 2 are many. We choose to mention those that arise naturally in geometry.

2.1. Let $M$ be a compact manifold on which a Lie group $G$ acts with cohomogeneity one (i.e., the principal orbits $G / H$ are of co-dimension 1). Then the orbit space $M / G$ is either a circle or an interval. In the latter case the two endpoints of the interval correspond to nonprincipal orbits $N_{1}, N_{2} \subset M$ and the manifold $M$ is obtained by gluing the normal disc bundles over $N_{1}$ and $N_{2}$ along their common boundaries.

We refer to $[4,5,7]$ for examples of manifolds admitting cohomogeneity one action of Lie groups.

2.2. Let $M$ be a Riemannian manifold that admits a transnormal function $f: M \rightarrow \mathbb{R}$ (i.e., there is a function $b: \mathbb{R} \rightarrow \mathbb{R}$ so that $\|d f\|^{2}=b(f)[\mathbf{1 0}]$ ), and let $N_{1}, N_{2} \subset M$ be the focal manifolds of $f$ corresponding to the maximum and minimum of $f$. Theorem A in [10] concludes that, again, $M$ is obtained by gluing the normal disc bundles over $N_{1}$ and $N_{2}$ along their common boundaries.

The idea of transnormal function is a natural generalization of the classical isoparametric functions, see $[\mathbf{1}, \mathbf{6}, \mathbf{1 0}]$.

2.3. Let $\pi: E \rightarrow M$ be a smooth fibration over a base manifold $M=D\left(\xi_{1}\right) \cup_{g}$ $D\left(\xi_{2}\right)$ having focal genus 2 . We set $E_{t}=\pi^{-1}\left(N_{t}\right)$ and put $\pi_{t}=\pi \mid E_{t}: E_{t} \rightarrow N_{t}$, $t \in\{1,2\}$. Then a focal genus 2 structure on $E$ with focal submanifolds $E_{t}$ is seen from

$$
E=D\left(\pi_{1}^{*} \xi_{1}\right) \cup_{G} D\left(\pi_{2}^{*} \xi_{2}\right),
$$

where $\pi_{t}^{*} \xi_{t}$ is the pullback of $\xi_{t}$ via $\pi_{t}$ and where the diffeomorphism $G$ is a bundle map over $g$.

2.4. Let $p: E \rightarrow S^{n}$ be a smooth fibration over the $n$-sphere $S^{n}$ with fiber $F$. A focal genus 2 structure on $E$ is afforded by

$$
E=F \times D^{n} \cup_{G} F \times D^{n}
$$

by 2.3, where the clutching diffeomorphism $G: F \times S^{n-1} \rightarrow F \times S^{n-1}$ has the form $G(z, v)=(\beta(v) z, v)$ for some map $\beta:\left(S^{n-1}, s_{0}\right) \rightarrow(\operatorname{Diff}(F), i d)$, and where $\operatorname{Diff}(F)$ is the group of diffeomorphism on $F$. It follows from $\beta\left(s_{0}\right)=i d$ that the corresponding homomorphism $\varphi$ in (3) acts identically on $H^{*}(F)$. It follows also from the triviality of $\xi_{2}$ that $e(\eta)=0$. Thus Theorem 1.1, together with Corollary 1.3, implies that

Corollary 2.1. Let $p: E \rightarrow S^{n}$ be a smooth fibration over the $n$-sphere $S^{n}$ with fiber $F$. There is an exact sequence

$$
\cdots \rightarrow H^{r-1}(F) \stackrel{\theta}{\rightarrow} H^{r-n}(F) \stackrel{\alpha}{\rightarrow} H^{r}(E) \stackrel{i^{*}}{\rightarrow} H^{r}(F) \stackrel{\theta}{\rightarrow} \cdots
$$

in which

i) $\alpha\left(i^{*}(x) \cup y\right)=x \cup \alpha(y)$;

ii) $\theta(x y)=\theta(x) y+(-1)^{(n-1) \operatorname{dim} x} x \theta(y)$.

In 1949 H.-C. Wang [9] studied fibrations $p: E \rightarrow S^{n}$ with the typical fiber $F$ a finite simplicial complex, and obtained an exact sequence (in analogue to (4)) in the homology groups of total space $E$ and the fiber $F$. The sequence (4) was later 
obtained as a consequence of the spectral sequence of a fibration and the property ii) was first observed by Leray $[\mathbf{2}$, p.442]. Property i) is also practical and a proof is given in $[\mathbf{1 1}$, p.336].

\section{Proof of Theorem 1.1}

For a smooth submanifold $i: N \hookrightarrow M$ in a Riemannian manifold $M$ with oriented normal bundle $\xi$, identify the unit disc bundle $D(\xi)$ of $\xi$ as a tubular neighborhood $t: D(\xi) \rightarrow M$ of the embedding. It induces the Excision isomorphism

$$
t^{*}: H^{*}(M, M \backslash N) \stackrel{\cong}{\rightrightarrows} H^{*}(D(\xi), D(\xi) \backslash N)
$$

and satisfies also $i=t \circ \sigma$, where $\sigma: N \rightarrow D(\xi)$ is the zero section. Since $\sigma$ is a homotopy inverse of the bundle projection $p: D(\xi) \rightarrow N$ we have

$$
t^{*}=p^{*} \circ i^{*}: H^{*}(M) \rightarrow H^{*}(D(\xi)) .
$$

Let $U \in H^{k}(D(\xi) ; D(\xi) \backslash N)$ be the Thom class of the oriented bundle $\xi, k=$ $\operatorname{dim}_{\mathbb{R}} \xi$. Cup product with $U$ yields the Thom isomorphism $[\mathbf{8}$, p.97]

$$
T: H^{*}(N) \underset{\cong}{\stackrel{p^{*}}{\rightrightarrows}} H^{*}(D(\xi)) \underset{\cong}{\stackrel{\cup U}{\rightrightarrows}} H^{*}(D(\xi) ; D(\xi) \backslash N), \quad T(\beta)=p^{*}(\beta) \cup U .
$$

Write $\psi$ for the composed isomorphism

$$
\psi=\left(t^{*}\right)^{-1} \circ T: H^{*}(N) \stackrel{\cong}{\rightrightarrows} H^{*}(M, M \backslash N)
$$

and let $s:(M, \emptyset) \rightarrow(M, M \backslash N)$ be the obvious inclusion of the topological pairs.

Lemma 3.1. For any $x \in H^{*}(M), y \in H^{*}(N)$ one has in $H^{*}(M)$ the relation

$$
x \cup s^{*} \psi(y)=s^{*} \psi\left(i^{*}(x) \cup y\right) .
$$

Proof. Consider the commutative diagram induced by $t$

$$
\begin{array}{ccc}
H^{*}(M) \times H^{*}(M, M \backslash N) & \stackrel{t^{*} \times t^{*}}{\longrightarrow} & H^{*}(D(\xi)) \times H^{*}(D(\xi) ; D(\xi) \backslash N) \\
\cup \downarrow & & \downarrow \cup \\
H^{*}(M, M \backslash N) & \stackrel{t^{*}}{\cong} & H^{*}(D(\xi) ; D(\xi) \backslash N)
\end{array}
$$

where the vertical maps are the cup products. It follows that for any $x \in H^{*}(M)$, $y \in H^{*}(N)$ one has in $H^{*}(D(\xi) ; D(\xi) \backslash N)$ that

$$
\begin{aligned}
t^{*}(x \cup \psi(y)) & =t^{*}(x) \cup t^{*}(\psi(y)) \\
& =\left(t^{*}(x) \cup p^{*}(y)\right) \cup U(\text { by the definition of } \psi) \\
& =p^{*}\left(i^{*}(x) \cup y\right) \cup U\left(\text { since } t^{*}=p^{*} \circ i^{*}\right) \\
& =T\left(i^{*}(x) \cup y\right)(\text { by the definition of } T) .
\end{aligned}
$$

Applying $s^{*} \circ t^{*-1}$ to the both ends of the equalities verifies Lemma 3.1.

Proof of Theorem 1.1. Let $M=D\left(\xi_{1}\right) \cup_{g} D\left(\xi_{2}\right)$ be a manifold having focal genus 2 and with focal submanifolds $i_{t}: N_{t} \hookrightarrow M, t=1,2$. Clearly, a tubular neighborhood of $i_{t}$ can be taken as $D\left(\xi_{t}\right) \subset M$. 
Assume that $t=1$ (consequently, $\bar{t}=2$ ) and that $\operatorname{dim} \xi_{2}=k$. Let

$$
j:(M, \emptyset) \rightarrow\left(M, N_{1}\right), h:\left(M, N_{1}\right) \rightarrow\left(M, M \backslash N_{2}\right)
$$

and $s:(M, \emptyset) \rightarrow\left(M, M \backslash N_{2}\right)$ be the obvious inclusions of the topological pairs. Since $N_{1}$ is a strong deformation retract of $M \backslash N_{2}$ the map $h$ induces an isomorphism on cohomology.

Define the map $\alpha_{1}$ by the commutativity of the diagram

$$
\begin{array}{ccc}
H^{r}\left(M, N_{1}\right) & \stackrel{j^{*}}{\rightarrow} & H^{r}(M) \\
h^{*} \uparrow \cong & s^{*} \nearrow & \uparrow \alpha_{1} \\
H^{r}\left(M, M \backslash N_{2}\right) & \stackrel{\psi}{\stackrel{\psi}{\cong}} & H^{r-k}\left(N_{2}\right)
\end{array},
$$

where the commutativity of the upper triangle follows from $s=h \circ j$. In view of the isomorphism $h^{*} \circ \psi$ we may replace in the cohomology exact sequence

$$
\cdots \rightarrow H^{r-1}\left(N_{1}\right) \stackrel{\partial}{\rightarrow} H^{r}\left(M, N_{1}\right) \stackrel{j^{*}}{\rightarrow} H^{r}(M) \stackrel{i_{1}^{*}}{\rightarrow} H^{r}\left(N_{1}\right) \rightarrow \cdots
$$

of the pair $\left(M, N_{1}\right)$ the relative cohomology group $H^{r}\left(M, N_{1}\right)$ by $H^{r-k}\left(N_{2}\right)$ to obtain

$$
\cdots \rightarrow H^{r-1}\left(N_{1}\right) \stackrel{\theta_{1}}{\rightarrow} H^{r-k}\left(N_{2}\right) \stackrel{\alpha_{1}}{\rightarrow} H^{r}(M) \stackrel{i_{1}^{*}}{\rightarrow} H^{r}\left(N_{1}\right) \rightarrow \cdots,
$$

where $\theta_{1}=\psi^{-1} \circ\left(h^{*}\right)^{-1} \circ \partial$. This establishes the exactness of the sequence (2).

Property iii) of the Theorem is essentially shown in the proof of [8, Theorem 11.3], and property i) comes directly from Lemma 3.1.

It remains to establish the decomposition ii) for the additive map $\theta_{1}=\psi^{-1}$ 。 $\left(h^{*}\right)^{-1} \circ \partial$ in (2). The inclusion $D\left(\xi_{2}\right) \hookrightarrow M$ gives rise to a relative homeomorphism $f:\left(D\left(\xi_{2}\right), S\left(\xi_{2}\right)\right) \hookrightarrow\left(M, D\left(\xi_{1}\right)\right)$ which satisfies $f \mid S\left(\xi_{2}\right)=g_{1}$ and induces also the (vertical) exact ladder

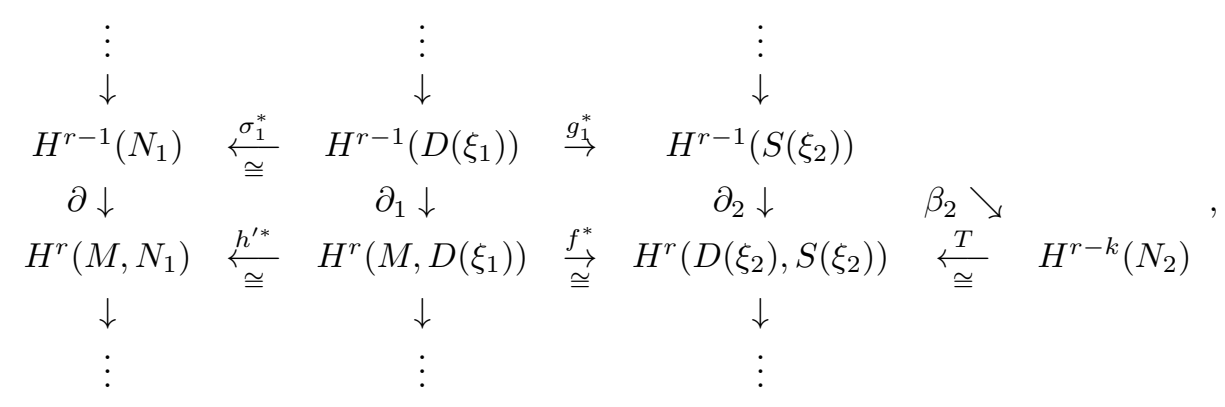

where $h^{\prime}:\left(M, N_{1}\right) \rightarrow\left(M, D\left(\xi_{1}\right)\right)$ is the obvious inclusion with $h^{\prime} \mid N_{1}=\sigma_{1}$, and where $\beta_{2}$ is the connecting homomorphism in the Gysin exact sequence of the sphere bundle $S\left(\xi_{2}\right) \rightarrow N_{2}$. Property ii) follows from commutativity of the above diagrams, as well as the following obvious relations

$$
\begin{aligned}
& g_{1}^{*} \circ\left(\sigma_{1}^{*}\right)^{-1}=g_{1}^{*} \circ p_{1}^{*} ; \\
& \theta_{1}=T^{-1} \circ f^{*} \circ\left(h^{\prime *}\right)^{-1} \circ \partial ; \\
& \beta_{2}=T^{-1} \circ \partial_{2},
\end{aligned}
$$

where $T$ is the Thom isomorphism of the bundle $\xi_{2}$. These complete the proof. 
HAIBAO DUAN

\section{References}

[1] U. Abresch, Isoparametric hypersurfaces with four or six distinct principal curvatures. Necessary conditions on the multiplicities, Math. Ann. 264 (1983), no. 3, 283-302.

[2] J. Dieudonné, A history of Algebraic and Differential Topology, 1900-1960, Boston, Basel, 1989.

[3] H. Duan, The degree of a Schubert variety, Adv. Math., 180(2003),112-133.

[4] K. Grove and S. Halperin, Dupin hypersurfaces, group actions and the double mapping cylinder, J. Differential Geom. Vol. 26, No. 3 (1987), 429-459.

[5] C. Hoelscher, Classification of cohomogeneity one manifolds in low dimensions, Ph.D. thesis, University of Pennsylvania, Philadelphia, 2007.

[6] W. Y. Hsiang, R. Palais and C. L. Terng, The topology of isoparametric submanifolds, J. Diff. Geom., Vol. 27 (1988), 423-460.

[7] A. Kollross, A classification of hyperpolar and cohomogeneity one actions, Trans. Amer. Math. Soc. 354 (2002), 571-612.

[8] J. Milnor and J. Stasheff, Characteristic classes, Ann. of Math. Studies 76, Princeton Univ. Press, 1975.

[9] H.-C. Wang, The homology groups of the fibre bundles over a sphere, Duke Math. J., 16(1949), 33-38

[10] Q. M. Wang, Isoparametric functions on Riemannian manifolds. I. Math. Ann. 277 (1987), no. 4, 639-646.

[11] G. W. Whitehead, Elements of homotopy theory. Graduate Texts in Mathematics, vol. 61, Springer-Verlag, 1978.

Haibao Duan dhb@math.ac.cn

Hua Loo-Keng Key Laboratory of Mathematics, Institute of Mathematics, Chinese Academy of Sciences, Beijing, 100190, P.R. China 